\title{
Two-Stage Surgery for a Large Cervical Dumbbell Tumour in Neurofibromatosis 1: A Case Report
}

\author{
Mohd Ariff S, MBBS, Joehaimey J*, M MED Orth, Ahmad Sabri 0*, M MED Orth \\ Abdul Halim $Y * *$, M MED Orth \\ Department of Orthopaedics, Traumatology and Rehabilitation, \\ International Islamic University Malaysia, Kuantan, Malaysia \\ *Department of Orthopaedics, Hospital Raja Perempuan Zainab II (HRPZII), Kota Bharu, Malaysia \\ **Department of Orthopaedics, School of Medical Sciences, Universiti Sains Malaysia, Kota Bharu, Malaysia
}

\begin{abstract}
Spinal neurofibromas occur sporadically and typically occur in association with neurofibromatosis 1. Patients afflicted with neurofibromatosis 1 usually present with involvement of several nerve roots. This report describes the case of a 14year-old child with a large intraspinal, but extradural tumour with paraspinal extension, dumbbell neurofibroma of the cervical region extending from the $\mathrm{C} 2$ to $\mathrm{C} 4$ vertebrae. The lesions were readily detected by MR imaging and were successfully resected in a two-stage surgery. The time interval between the first and second surgery was one month. We provide a brief review of the literature regarding various surgical approaches, emphasising the utility of anterior and posterior approaches.
\end{abstract}

Key Words:

Neurofibromatosis 1, Dumbbell Neurofibroma, Spinal Tumour, Cervical Spine, Surgical Approach

\section{INTRODUCTION}

Neurofibromatosis is a common, multisystemic, autosomal dominant disorder in which affected individuals develop both benign and malignant tumours. Two distinct forms have been recognized based on clinical and genetic criteria. Neurofibromatosis ${ }^{1}$ (NF-1; von Recklinghausen's disease; peripheral type) accounts for $85 \%$ of all neurofibromatosis patients and very commonly involves the musculoskeletal system ${ }^{1}$. Unlike NF-1, NF-2 (central type) rarely includes orthopaedic complications. The USA National Institutes of Health has developed consensus criteria for the diagnosis of both disease types ${ }^{2}$.

Neurofibromas frequently show concentric enlargement along the spinal nerve. The term "dumbbell tumour" describes spinal tumours that acquire an hourglass shape in the course of growth, as anatomic barriers such as the dura mater, nerve root foramen, or bony elements are encountered ${ }^{1}$. Resection raises several problems, including the risk of general anaesthesia, preservation of the spinal cord and/ or nerve root, control of the vertebral artery, and maintenance of spinal motion and stability ${ }^{1,3}$. Here, we present the case of a 14-year-old child with a large dumbbell neurofibroma of the cervical region and the two-stage tumour resection that was successfully accomplished.

\section{CASE REPORT}

A 14-year-old boy with NF-1 was referred to us for a unilateral neck swelling associated with progressive quadriparesis. One month prior to admission, he noticed a right-sided neck swelling that progressively increased in size. Simultaneously, he experienced increasing difficulty in walking. There were no constitutional symptoms. He was a child of a non-consanguineous marriage with positive family history for von Recklinghausen's disease.

On examination, the patient had multiple cafe-au-lait spots. There was a severe cervical compressive myelopathy with upper motor neuron lesion symptoms from C3 downward. A $7 \times 5 \mathrm{~cm}$ mass was noted in the right submandibular region that was firm-to-hard in consistency.

MR images revealed a large intraspinal but extradural tumour on the right side, extending from $\mathrm{C} 2$ to $\mathrm{C} 4$, displacing and compressing the spinal cord to the left. The growth extended through $\mathrm{C} 2 / \mathrm{C} 3$ and $\mathrm{C} 3 / \mathrm{C} 4$ exit foramina causing widening of the foramina and a lobulated component in the neck (Figure 1). Features were suggestive of a C2-C4 nerve sheath tumour with spinal cord compression.

Total laminectomy and unilateral facetectomy of C3 were performed, with preservation of supraspinous and interspinous ligaments of $\mathrm{C} 3-\mathrm{C} 4$ segment. This was 


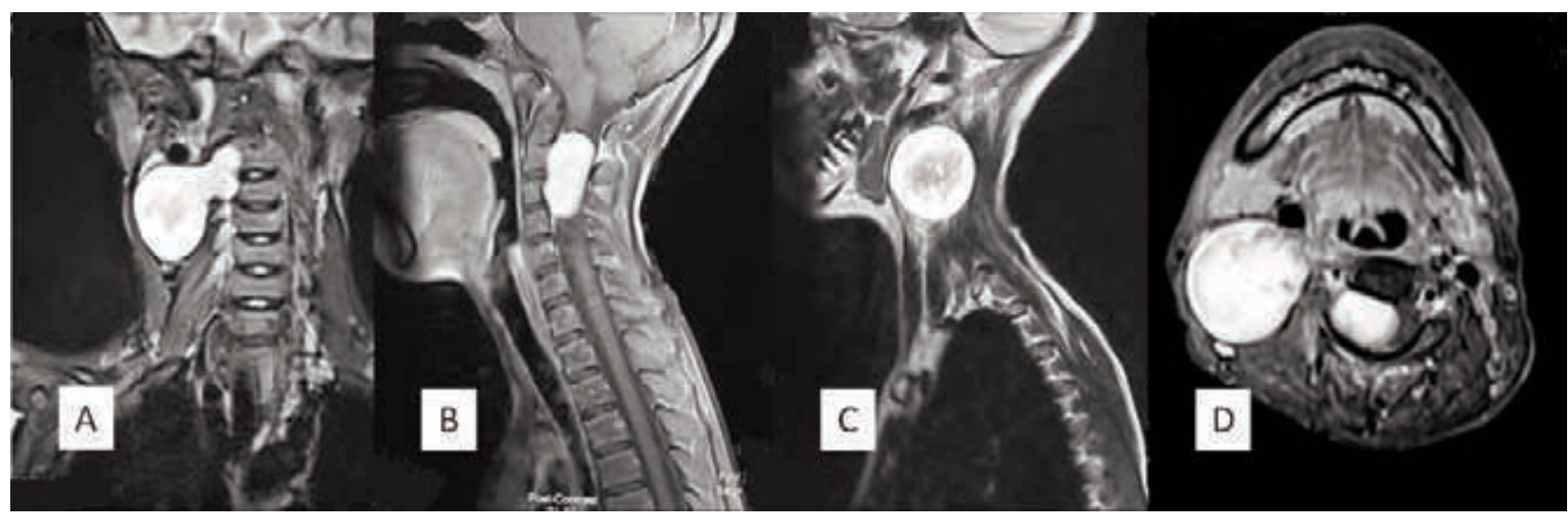

Fig. 1: $\quad$ MRI of the cervical spine (A) Coronal T1-weighted image showing both intra- and extraspinal tumour. (B) Sagittal T2-weighted image showing intraspinal tumour almost completely obstructing the spinal canal. (C) Large extraspinal tumour on T1-weighted image. (D) Axial view with intraspinal tumour displacing and compressing the spinal cord to the left.
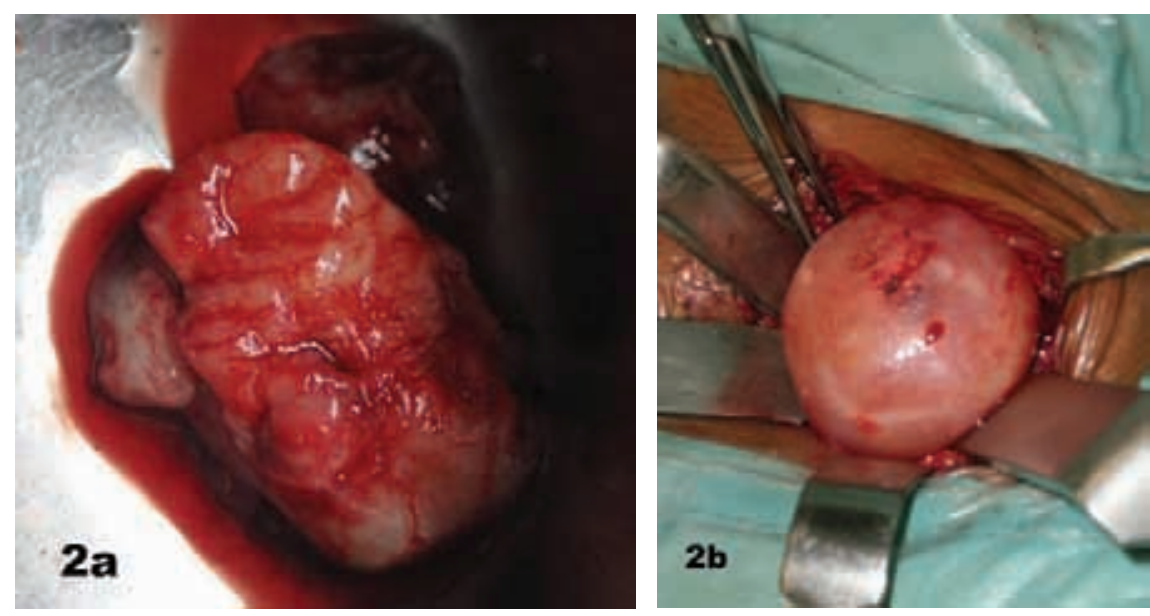

Fig. 2: Intra-operative picture showing (A) intraspinal tumour after resection, and (B) extraspinal tumour resection via anterior approach.

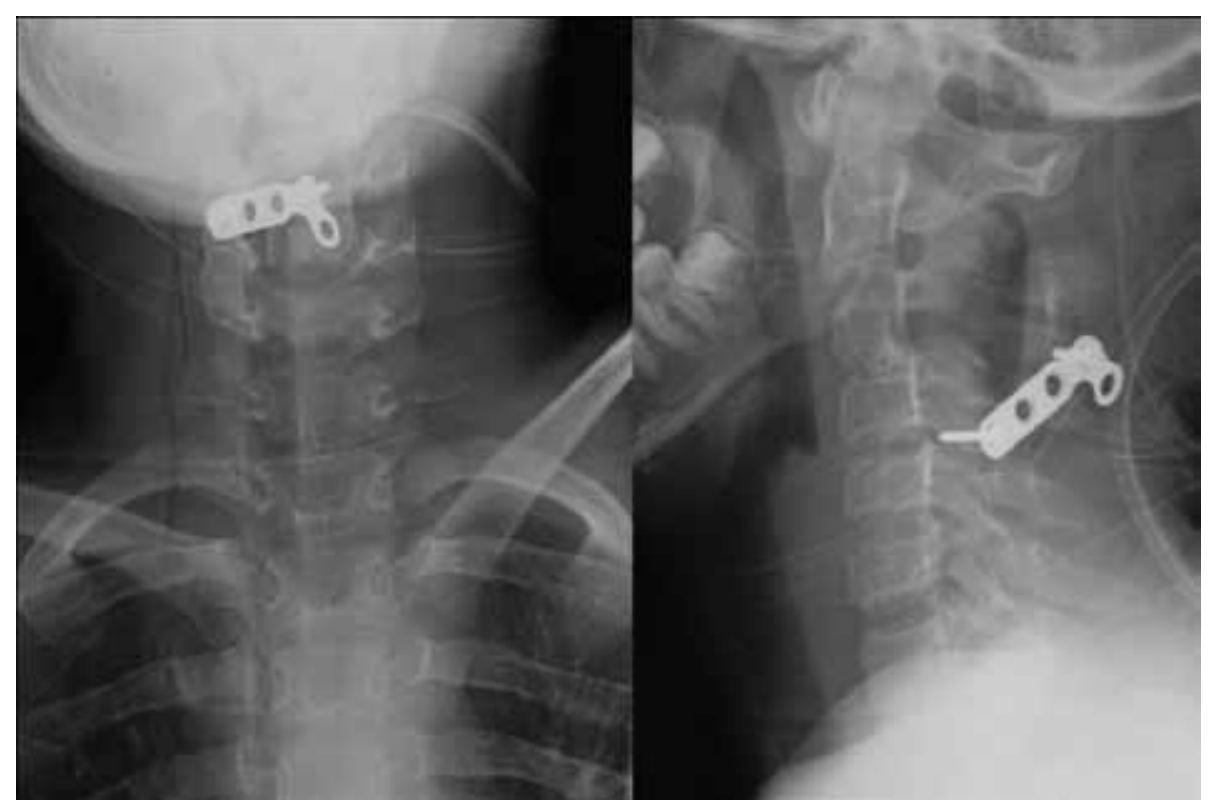

Fig. 3: Plain radiographs of the cervical spine six month post surgery. 
accomplished by horizontally resecting the tip of the $\mathrm{C} 3$ spinous process and retracting laterally. Intraoperatively, we noted that approximately $90 \%$ of the spinal canal was occupied by the tumour. The intraspinal component was almost entirely resected (Figure 2a) as well as the right $\mathrm{C} 3$ nerve root; other involved nerve roots were preserved. A 4hole L-mini-plate was fixed at the $\mathrm{C} 4$ spinous process to the facet joint of C4-C5 to avoid collapse of the destabilized segment. Estimated blood loss was about two litres. The patient showed dramatic recovery immediately following surgery. He was able to walk with the assistance of one person and feed himself with a spoon within the first week. The patient was discharged to await the follow-up surgery (one month after the first surgery). During the second surgical procedure, the extraspinal component in the neck and remnant of the tumour at the intervertebral foramen were resected via an anterior approach (Figure 2b). The surgery was uneventful. Following the second surgery, neurological recovery further improved. By the end of the second week, the patient functioned independently, and also independently performed fine motor functions such as writing. Histopathological diagnosis of the resected specimens showed neurofibromas.

Six months following surgery, the patient had no complaints of neck symptoms and no recurrence of neurological deficit. Plain radiographs of the cervical spine showed neither instability nor abnormal alignment (Figure 3). Currently the patient is able to independently carry out activities of daily living. He is still being followed up for any recurrence or late onset complications.

\section{DISCUSSION}

Surgical treatment of cervical dumbbell neurofibromas is highly successful due to the benign nature of the tumours ${ }^{3,4}$. However, these procedures can be challenging, and may be associated with numerous perioperative complications. Related major unsolved problems include preservation of involved spinal roots, high recurrence rate and postoperative cervical deformity ${ }^{3}$. The ideal surgical strategy will be to achieve complete removal to reduce the risk of recurrence, while at the same time providing adequate stability to avoid cervical deformity.

Large tumours with significant extraspinal extension frequently require combined anterior and posterior exposure for successful and complete removal ${ }^{4,5}$. This approach can have disadvantages, prolonged surgical time and more extensive blood loss. Some authors advocate a lateral approach, which provides extensive exposure, and does not require repositioning of the patient. It remains, however, a time-consuming and technically demanding procedure ${ }^{5}$, and still carries the risk of injuring the surrounding vital structures, including the vertebral arteries and surrounding nerves.
Onesti et $a^{5}$ reported their experience using a transparaspinal approach that combines laminectomy and sectioning of the paraspinal muscles through a transverse incision. This approach is appropriate for use over the entire spine, and there is no upper limit of tumour size for which it is applicable. It is a simpler and quicker technique, and provides excellent exposure of the lateral aspects of the spine, facilitating complete removal of paraspinal tumours without the need for subsequent anterior surgery. This approach is contraindicated for tumours with significant anterior spinal cord compression, as it does not permit adequate visualization of the anterior dural margin. Anterior column reconstruction is also not possible in such cases.

Jiang et al developed a practical classification system (Peking University Third Hospital, PUTH) for determining the appropriate surgical approach for cervical dumbbell tumour resection based on pre-operative MRI and/or CT images ${ }^{4}$. The PUTH classification is based on tumour type and anatomic locations. They recommend a posterior approach with facetectomy or an anterolateral approach for tumours similar to the case described here.

We chose a two-stage approach, the first stage to provide extensive intraspinal access posteriorly and a second procedure to complete resection of the large, extraspinal extension via the anterior exposure. In this case, prognosis was good despite the severity of preoperative neurological deficit. The two-stage procedure with a longer interval between stages provides a safe surgery and decreases intraoperative morbidity to the patient, due to avoidance of prolonged operative time and massive blood loss. A longer interval also provides time for the initial surgical wound to heal and for oedema absorption around the spinal cord from the first surgery.

Benign cervical neuromas have a high recurrence rate $(10.7 \% \text { at } 5 \text { years and } 28.2 \% \text { after } 10 \text { to } 15 \text { years })^{3}$. Preservation of the involved nerve roots should always be a priority; however, it is sometimes difficult or even impossible to achieve radical resection, a factor that contributes to the high recurrence rate. Patients remain asymptomatic in most cases, even in recurrence of NF-1. Furthermore, the recurrence rate is significantly higher in NF-2 despite the high ratio of completely resected tumours ${ }^{3}$. Cervical instability has been reported in $20 \%$ of patients following posterior approach procedures. Jiang et al reported that patients who undergo facetectomy without fusion have a $50 \%$ risk of scoliosis or kyphoscoliosis, with or without neurological deficit ${ }^{4}$. The authors recommend that fixation be performed for all cases in order to prevent such complication, especially for posterior revision cases. 


\section{REFERENCES}

1. Hirsch NP, Murphy A, Radcliffe JJ. Neurofibromatosis: clinical presentations and anaesthetic implications. Br J Anaesth. 2001; 86(4): 555-64.

2. Gutmann DH, Aylsworth A, Carey JC, Korf B, Marks J, Pyeritz RE, et al. The diagnostic evaluation and multidisciplinary management of neurofibromatosis 1 and neurofibromatosis 2. JAMA. 1997; 278(1): 51-7.

3. Klekamp J, Samii M. Surgery of spinal nerve sheath tumors with special reference to neurofibromatosis. Neurosurgery. 1998; 42(2): 279-90.

4. Jiang L, Lv Y, Liu XG, Ma QJ, Wei F, Dang GT, et al. Results of surgical treatment of cervical dumbbell tumors: surgical approach and development of an anatomic classification system. Spine. 2009; 34(12): 1307-14.

5. Onesti ST, Ashkenazi E, Michelsen WJ. Transparaspinal exposure of dumbbell tumors of the spine. J Neurosurg. 1998; 88(1): 106-10. 